\title{
Study of the Bionic Humanoid Robot in Industrial Design
}

\author{
Wang xinwei ${ }^{1, a}$, Lv desheng ${ }^{2, b^{*}}$ \\ ${ }^{1}$ Harbin Institute of Technology, 92 West Dazhi Street, Harbin 150001, China \\ ${ }^{2}$ Harbin Institute of Technology, 92 West Dazhi Street, Harbin 150001, China \\ axinwei0932@163.com , bdeshengl@hit.edu.cn
}

\section{Keywords: Bionic Design; Humanoid Robot; Industrial Design; Intelligent Control}

\begin{abstract}
The main features of product design embodies in the diversity and singularity of ecological material form, nature is a rich design library, nature gave birth to a variety of species, every creature has magical modeling characteristics and biological characteristics, these vivid characteristics constitute the nature's most distinctive ecological law. This is the place that humanoid robot bionic design derived, with multi-faceted imitation and reference to characters in the design of robot, endowing the product with natural characteristics, based on the concept and application field of characters form bionic, from the perspective of modeling, functioning and mechanical structure. The article proposed the concept and applications of bionic, exploring its numerous features, the broad prospects of development brought by the control of intelligent information and highly active adsorption material bionic, their revelation of industrial design, all provided new ideas and methods for industrial design, playing an important role in the development of industrial design.
\end{abstract}

\section{Introduction}

Since ancient times, nature is the foundation of human survival, meanwhile, as well as transformation of human beings. The nature is very rich in species, each creature has its own form, color, function, structure and biological activities. Through researches and observations of natural systems, human refined inspiration of invent from the extraction of natural law. The sophisticated organism structure, the perfect functions, the scientific movement principles and biological behaviors have been imitated and used by robot designers. Bionic robot design is a combination of science and art, and is also a product of harmonious coexistence between man and nature the method of bionic design will certainly help to design more excellent bionic robot works.

\section{Bionic of humanoid robot.}

Bionics is an independent discipline, it is a science mainly by simulating biological principles to build technical system or to make artificial technology system similar to the biological characteristics.[1] Bionic robot design is the extension and development of bionics, it is a design method mainly through the study of human main external morphological structure characteristics and the cognition of its symbolic meaning, based on the original form, the understanding and application of the nature of morphological in order to simulate, process and re-invent. It mainly studies the design relationship between designer and bionic robot, taking human as the basic elements of the design, digs out more creative origin from observation and understanding of characters shape, simplifies and refines external characteristics and internal structures, expresses by using art performances, such as deformation, abstract and exaggeration. The designers are given full play to their imagination; at the same time they also should combine the characteristics of the existing technology and development of intelligent technology, in focusing on the arts while also combining science and technology to achieve the perfect combination of art and technology. Thus to match the bionic robot art connotation and to realize its real value, and more robots modeling design will be created combined with the humanized design of bionic 
design, usability design, emotional design and other related design concepts, and the integration between man and nature, man and science will also be achieved.

In the evolution process of billions of biological organisms, their internal structures, external forms and motor functions have all been greatly improved and optimized, and may have further development and improvement in the future. With the in-depth study of bionics, the application of bionic design began to step into the research and development of humanoid robot. Now humanoid robots are used in many aspects and fields, such as aerospace, military, medical, disaster relief and emergency, etc. Mainly to help mankind to explore the unknown and to complete difficult tasks instead of human. But with a broader learning ability and perception of mobile space, bionic humanoid robot has more advantages; it can not only walk on the ground and a variety of terrain but also identify small blind spots. The application of bionic design allows great improvement of the modeling of humanoid robot has been greatly improved, maintains the aesthetic needs of people, truly combines human beings and nature, nature and technology which are one of the design development trends in future humanoid robot.

\section{Biomimicry in design humanoid robot.}

With the deepening of the understanding of human nature, it was found that bionic designs on plants and animals have been far from satisfying the demand of human beings. Compared to other industrial robot design, humanoid robot design has many advantages and can adapt to a wide variety of human life and working environment, in many ways it even can go beyond human instinct. When the development of science and technology was not yet fully mature, humanoid robot designed only stayed on the basis of macroscopic and was relatively simple, there was no real combination of technology and aesthetics. But through the understanding reflection of their own functions and structures, coupled with the arrival of intelligent design, the design of robot is not just to imitate human appearance but also through the study of internal structures, functions, emotions, perceptions and learning abilities, a mobile intelligent robot with awareness function, operating function, learning ability, self-control, associative memory and emotional interaction can be created by applying them to the design . [2] It realizes the real meaning of robot design and opens future development path and direction of humanoid robot design.

Shape bionic humanoid robot design. Form bionics is the most common way of humanoid robot designs. Designers learn from nature and extract bionic materials from it. By accumulation, transformation, innovation, they can make product modeling diverse, delight, epoch and the robot form affination.

Humanoid robot bionic form design is mainly on extraction, initiation, deformation of the whole human form, to achieve the purpose of design through scientific analysis. The main object of study including head, body, arms, hands, legs and other external features and its relevance, and will be applied in the design of humanoid robot through the art of visual aesthetic feeling and symbolic processing technique. At the same time, ergonomics, mechanics, materials, and other related aspects of intelligence are fully integrated.

Form design is a primary consideration in product design, bearing the designer's design concepts and design ideas. Natural form design is the basis and driving force of modeling design. All things in nature are beautiful, have their existence values, extracting beautiful things can create more beautiful shapes and varieties of bionic product designs. Humanoid robot design has the history of several decades, and now has become main research directions in the field of robotics research. In 1968, Smosher ,a US General Electric Company researcher developed a manipulation type two-legged walking mechanism named Rig, which was the prelude of humanoid robot research. [3] In 1968, Professor Ichiro Kato in Japan first started working on humanoid robot, and had successfully developed WL-5 type two-legged walking robot with 11 degrees of freedom in 1971. With the advancement of research, Honda 
Company had developed a series of humanoid robots, from the first generation E0 to new robots "ASIMO" (refer with: Fig. 1). [4] As can be seen from the figure the early humanoid model design is simple, is the abstract bionic of human form, emphasized on local bionic modeling design of and gives a sense of cold. At that time the design is mainly on technology, ignoring visual effect, it is not the real combination of technology and aesthetics, thus the appearance is indifference, not affinity. ASIMO applied the extracted the main exterior characteristics of humanoid appearance to humanoid robot design, which shows local bionic design extends to overall bionic design, abstract bionic design of transits to representational bionic design, including proportion structure of human body .

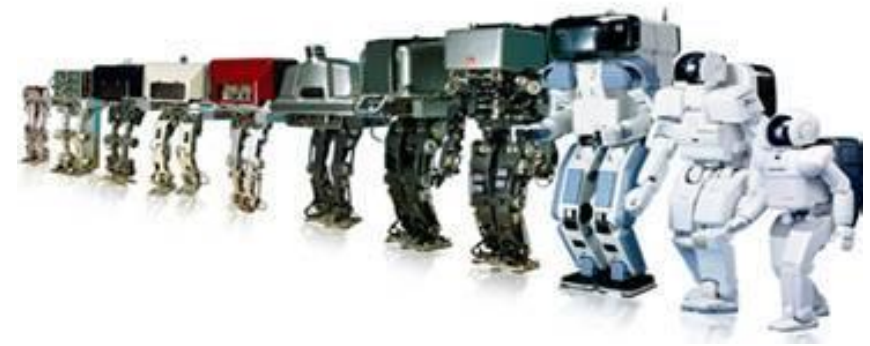

Figure. 1 ASIMO's evolution

Functional bionic design. For the continuous improvement of our own special function 、 the physical mechanism and language communication. Intelligent robot language communication function will be more and more perfect in the future. It can grasp a number of language and beyond the human ability to learn. [5]Humans are evolved in the development under severe nature environment, thus survive in natural environment. To some extent the modern functional bionic design is mainly implemented by means of science and technology, and verifies the validity, reliability and practicability of the function through continuous scientific experiment.

In recent years, research and development of humanoid robots has aroused wide attention of the scientific community, imitation increased from local human function to the whole body including facial expressions and emotions. With the help of functional bionic people can complete single task in a specific environment. In some dangerous and complex environment which requires human participation, such as pinch, pull, pull, pull, tearing, grinding and other actions, it appears the weakness of general industrial robots. Five fingers dexterous hand, is like a hand with five fingers, a palm touch and tactile function distribution, has strong functions and high universality,[6] this is the imitation of human fingers function. It can replace and help human with complex tasks. For example, ASIMO, It can be very natural to do all kinds of complicated movements, such up and down the stairs, shake hands with people, hold water, dancing and playing, etc.[7] Functions of human body are endless, careful observation and trial and error can help us design more bionic functions.

Humanoid robot Structural Design. In industrial design, designers study the overall structure of the human body through structural knowledge of the human body from the inside out, including human body, muscle and bone structure. Structural similarity imitation is according to different uses of robots, and is applied to the design of bionic robots. Structural design should not only focus on the effect of bionic robot, but also pay attention to aesthetic value of robot structure, it real application of man-machine structure to aesthetics.

Taking bionic robot arm as an example, bionic arm imitation is the most important feature of a humanoid robot, to understand the humanoid robot arm, first mechanisms characteristics of human arm are needed to understand. Human arm consists of shoulder, arm, elbow, forearm, wrist, hand and other components.[8] According to the reality of humanoid and movement of the robot, we now develop a total of nine degrees of freedom, among them, the shoulder joint of two degrees of freedom, elbow belongs to single shaft joint, with one degree of freedom, wrist joint one degree of freedom, the head pitching one degree of freedom. 
Humanoid robot body part has a role in connecting arms, head and legs, and installation space must be left, similar to the human shape, the humanoid robot shoulder motor box using the rectangular box, with one on each side, both sides of each motor box has 4 screw holes, covered in 2 boards on the side of the motor box, with a screw, then the humanoid robot's body formed. The same to arm part of rectangular box and connection plate thus formed the entire arm. (refer with: Fig. 2).

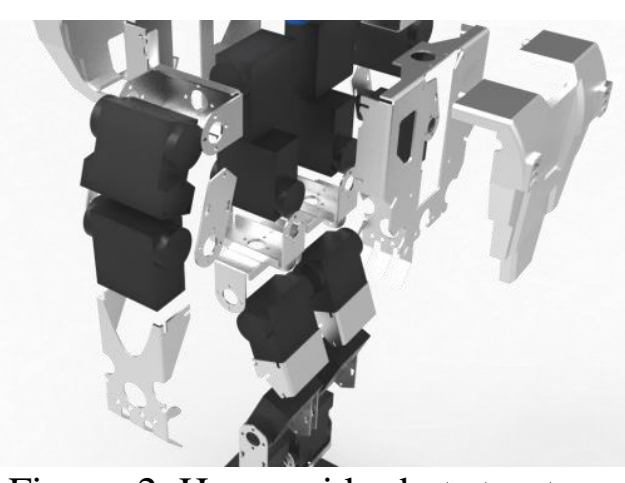

Figure. 2 Humanoid robot structure

\section{The robot bionics development of philosophy and revelation.}

Modern scientific progress and development, have broaden people's horizons and capabilities of aesthetic. Bionics robot is developed on the basis of the continuous improvement in the material and spiritual life of society. Robot bionics in industrial Design is an interdisciplinary, which combines aesthetics, art, science, materials and artificial intelligence, and focuses on imitating nature and emotional design in the development of bionic design concept. This gives industrial designers new inspirations and enlightenment in finding a harmonious relationship between humans and nature, humanity and emotion, humans and robots.

Intelligent control of bionic cut a figure. Applications of intelligent humanoid robot bionics is more extensive, information and control bionic is one of the important areas, on the one hand it is the development requirement of manual to intelligent control, on the other hand it is the needs and challenges of biomimetic robot development. In 2012, "Robot Story" with smooth robot movements, perfect cooperation light and music, stood out in so many wonderful programs, impressed the audience and gained laughter and applause (refer with: Fig. 3). Spring Festival gala robot can separate display, as well as the collective performance of street dance, gymnastics and plays to show robot flexible and intelligent.

People get the high-end technology in the field of robotics when they enjoy the joy brought by the show. it is more important to draw the audience's interest to the development of science and technology, promote the strategy relying on science and education, it is one of the most ideal elements to close the distance between audience and robots. Dancing robot should be made not only robust and reliable, but also having a flexible action, thus it can achieve synchronization with music. The dancing of robots has been widespread concern as an art combining of auditory and visual arts.

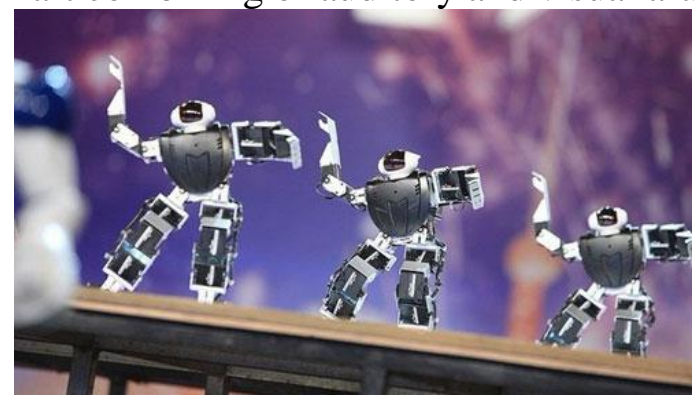

Figure. 3 The“robot story" 
Broad prospects of high active bionic material adsorption. The rapid development of the robot forces engineers and designers to work hard in the design, in order to create a "robot of extreme simulation". In 2010, Japanese roboticist Hiroshi Ishiguro created a female robot called" Geminoid F "[9], the skin of the robot is mainly made of soft silicone, of no distinction with real people over a long distance.[10] The rich facial expressions of eyes and mouth movements, fluency of speech based on dialogue recognition, human-computer interaction on the basis of machine vision, all make people feel it as real one. The personification robot developed by this robot research institute, is with the same reality shape, its main body parts can pose a variety of gestures and movements, and it is able to interact with people by using simple and flexible body movements, combining with advanced speech recognition technology. The relationship between machine and human being becomes the communication between peoples, and has a part of human intelligence and over-human intelligence, makes the bionic robot effect to achieve perfection, and gradually move closer to the simulation technology, these are development results of silicone bionic robot.

\section{Summary}

With further research in the field of bionics and robotics technology, human longing and yearning for a return to nature, the using of characters form bionic thinking to redesign, re-creation is the result of scientific development, as well as the combination of industrial design activities and nature. Making human society to achieve harmonious coexistence with nature is a very important design method. According with the demand of sustainable development of human and the demand of the harmonious coexistence with nature, characters form bionics is gradually becoming a new bright spot and a new direction in the development of industrial design, greatly enriching the design idea and method of industrial design and emotional needs, reaching a high degree of unity between man and nature.

\section{Acknowledgements}

This research is funded by Heilongjiang Provincial Natural Science Funds of China (Grant NO.: LC2013C19).

\section{References}

[1] M.D.Luo: China Science and Technology Information, (1998) No.21,p.28-29. In Chinese.

[2] J.Y.Wang, J.S.Wang, J.J.Zhang: Machinery Design and Manufacture, DOI:10.3969/j.issn.10013997.2012.09.056. (2012)No.9,p.156-158. In Chinese.

[3] J.J.Xi: Intelligent biped robot control and collaborative research (MS, Xidian University,China 2012),p.2. In Chinese.

[4] J.Chestnutt, M.Lau, G.Cheung: Proceedings of the 2005 IEEE International Conference (IEEE, ICRA, 2005),p.629-634.

Reference to three books:

[5] G.M.Xie, X.Z.Kong, C.G.He:Overview of the robot(Harbin Engineering University Press China 2013),p.7. In Chinese.

[6] N.F.Xiao:Humanoid Robot(Science Press China 2008),p.1-10. In Chinese.

[7] S.H.Piao: Intelligent Robot( Harbin Institute of Technology Press China 2011),p.6. In Chinese.

[8] Z.L.Wang, B.C.Zhang,Z.X.Pang: Machinery Design and Manufacture, DOI:10.3969/j.issn.1001- 
3997.2011.07.015. ( 2011) No.7,p.38-40. In Chinese.

[9]C.Becker-Asano,H.Ishiguro: Affective Computational Intelligence (WACI), 2011 IEEE Workshop( IEEE, 2011),p.1 - 8.

[10] Information on http://baike.baidu.com/view/4748712.htm 